\title{
Visual Design of Physical User Interfaces for NFC-based Mobile Interaction
}

\author{
Alina Hang \\ Media Informatics Group, \\ University of Munich (LMU) \\ Munich, Germany \\ alina.hang@ifi.lmu.de
}

\author{
Gregor Broll \\ DOCOMO Euro-Labs \\ Munich, Germany \\ broll@docomolab-euro.com
}

\author{
Alexander Wiethoff \\ Media Informatics Group, \\ University of Munich (LMU) \\ Munich, Germany \\ alexander.wiethoff@ifi.lmu.de
}

\begin{abstract}
Near Field Communication (NFC) can facilitate mobile interaction with everyday objects, associated digital information and ubiquitous services. Despite the simplicity of the touch-like interaction between mobile devices and tagged physical objects, most people are still unfamiliar with this physical interaction, resulting in various usability problems. In order to improve mobile interaction with NFC-tagged objects, that serve as physical user interfaces (UI), we examine various aspects of their visual design. We identify different phases of mobile interaction with tagged objects and apply a user centered design process to create and evaluate different symbols for these phases. We report on the iterative design of NFC-symbols and physical UIs using low- and high-fidelity prototyping and present the results of a user study which was carried out with an experience prototype for an advertising scenario.
\end{abstract}

\section{Keywords}

Near Field Communication, physical user interfaces, visual design, low-fidelity prototyping, experience prototype

\section{ACM Classification Keywords}

H.5.2 [Information Interfaces and Presentation]: User Interfaces - prototyping, user-centered design

\section{INTRODUCTION}

Near Field Communication (NFC) is an emerging radiobased technology for the contactless exchange of data over short distances [27]. It stores digital information on passive, wireless tags that can be attached to everyday objects. Users can retrieve information from tags by simply touching them with a reading device, e.g. an NFC-enabled mobile phone, or by holding them closely together. An increasing number of applications take advantage of the simplicity and convenience of this physical interaction to facilitate payment, ticketing, identification, access control or information retrieval. Analysts estimate that by 2012, more than $20 \%$ of all mobile devices will include NFC [1].

Permission to make digital or hard copies of all or part of this work for personal or classroom use is granted without fee provided that copies are not made or distributed for profit or commercial advantage and that copies bear this notice and the full citation on the first page. To copy otherwise, to republish, to post on servers or to redistribute to lists, requires prior specific permission and/or a fee.

DIS 2010, August 16-20, 2010, Aarhus Denmark

Copyright (C) 2010 ACM ISBN 978-1-4503-0103-9, 2010/08 - \$10.00.
NFC can facilitate mobile interaction with tagged objects, associated information and ubiquitous services in different ways: NFC-tags can serve as physical hyperlinks [22] that reduce complex interactions to the simple touching of a single tag, e.g. to open a website. Tagged objects like posters, leaflets or billboards can serve as physical user interfaces (UI) that comprise multiple tags to adopt features of mobile UIs and to allow more elaborate NFC-based interactions with them. Examples include posters for mobile ticketing [4], menus for home delivery [11] or multimedia player controls [21].

Due to the novelty of NFC and its low dissemination outside of eastern Asia, most people are not yet familiar with this new technology and hardly know about it. Upon contact with NFC-tags and interactive physical objects, they often face an inhibition threshold, do not know how to carry out the interaction with NFC and make various mistakes [3, 4]. Opposite to visual markers which are clearly recognizable, NFC-tags are often integrated with physical objects and less visually present. Studies have shown that users do not exhibit a clear mental model about NFC in means of a common brand [8, 14]. Although the NFC Forum has introduced a trademark for NFC in $2009^{1}$, existing applications use different proprietary symbols (Figure 1) which decreases the recognition value of NFC.

This situation shows the need for a common visual language for NFC-based mobile interactions in order to increase the recognition value of NFC, to establish a mental model about its usage and to explain the interaction with it. Therefore, the goal of this paper is to investigate the visual design of physical UIs for NFC-based mobile interactions. This includes the accentuation of tagged objects and single tags as interactive elements, the explanation of their different functionalities or the design of complete physical UIs. Improving the visual design of NFC-based mobile interactions can help users to become more familiar with this new technology, facilitate the initial contact with it, make the interactions more comprehensible, prevent errors or provide better guidance during the interaction process.

The next section gives an overview of related work on NFC-based mobile interaction. Sections 3 and 4 outline the design process of this paper and describe different phases

\footnotetext{
${ }^{1}$ www.nfc-forum.org/resources/N-Mark
} 
of NFC-based mobile interaction in order to provide the conceptual background for the subsequent sections. Following the design process, sections 5 and 6 present lowand high-fidelity prototypes of different visual designs for NFC-symbols and physical UIs in order to support different phases of the interaction process. Based on this prototyping, section 7 presents the evaluation of an experience prototype for an interactive poster. Section 8 discusses the results and concludes this paper.

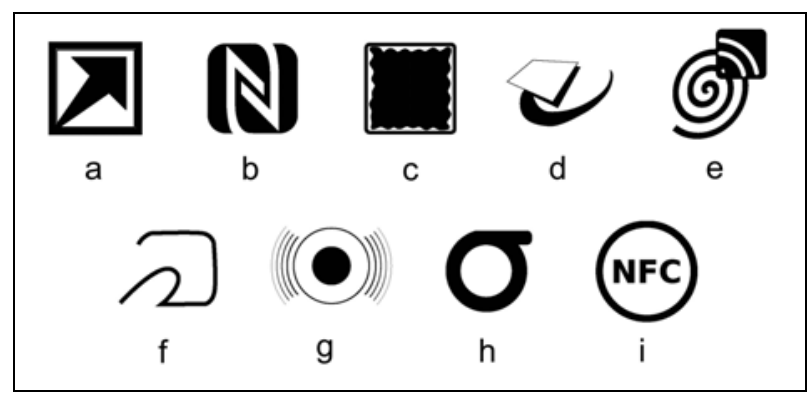

Figure 1. Sketches of existing logos and symbols for NFC: NFC-trial in Caen ${ }^{2}$ (a), NFC Forum N-Mark ${ }^{1}$ (b), Violet Ztamp:s ${ }^{3}$ (c), Oyster Card ${ }^{4}$ (d), Nokia 3220 NFC $^{5}$ (e), Sony Felica ${ }^{6}$ (f), DB Touch\& Travel ${ }^{7}$ (g), Alcatel Lucent touchatag $^{8}(\mathrm{~h})$ and Selecta ${ }^{9}$ (i)

\section{RELATED WORK}

NFC and the related Radio Frequency Identification (RFID) [27] have gained a lot of interest as technologies for short-range, contactless data exchange with smartcards, mobile devices and wireless tags. An increasing number of (commercial) applications use them to facilitate mobile interactions, especially ticketing (e.g. i-mode FeliCa ${ }^{10}$ ) and payment (e.g. Oyster Card ${ }^{4}$ ). Mobilkom Austria offers payment and ticketing with NFC-enabled mobile phones ${ }^{11}$. Trials for mobile ticketing with NFC have been conducted by Deutsche Bahn (Touch and Travel ${ }^{7}$ ) and Rhein-MainVerkehrsverbund $^{12}$ in Germany. The SmartTouch ${ }^{13}$ project has investigated NFC in different use cases, such as ticketing, access control, home care or entertainment. The Internet of Things [12] uses RFID-tags to attach unique Electronic Product Codes (EPC) to objects in order to identify them on a network, e.g. for logistics.

\footnotetext{
2 www.nxp.com/news/content/file_1193.html

3 www.violet.net

${ }^{4}$ https://oyster.tfl.gov.uk/oyster/entry.do

5 www.forum.nokia.com/devices/3220/

${ }^{6}$ www.sony.net/Products/felica/

7 www.touchandtravel.de

8 www.touchatag.com/

9 www.at.selecta.com

10 www.nttdocomo.co.jp/english/service/imode/make/content/ felica/index.html

11 www.mobilkom.at/de/nfc

12 www.rmv.de/coremedia/generator/RMV/Tickets/RMVHandyTicket

13 www.smarttouch.org
}

In Ubicomp research, RFID and NFC are enabling technologies for mobile interactions with tagged objects and associated digital resources. In 1999, Want et al. [26] already linked everyday objects (e.g. books, documents, business cards) and digital resources (e.g. electronic documents, URLs, email-addresses) through RFID-tags. While most applications use single tags as physical hyperlinks, other ones realize more complex NFC-based mobile interactions with elaborate physical UIs and multiple tags. The PERCI-project [4] has developed some of the first examples for smart posters that comprise multiple tags to let users invoke Web Services for mobile ticketing. Sanchez et al. [21] use physical UIs to operate media-players whose control commands have been implemented with RFID-tags. In [11], the authors present a home care service that allows elderly people to order meals for home delivery by touching RFID-tags on a menu with their mobile phones. Hardy et al. [9] use a grid of NFC-tags as an interactive surface for applications whose GUI is projected onto the grid. The mobile phone is used to select tags on the grid and to manipulate the projected interface.

Despite the increasing dissemination of NFC, most people are still unfamiliar with this new technology. Previous studies have uncovered recurrent usability problems during NFC-based mobile interaction that motivate a better visual design: For example, it is difficult for novice users to identify the interactive elements on physical UIs, even though they are often marked with a special symbol for NFC [4]. This may be because the symbols are not yet widely known, vary depending on the application (Figure 1) or because they are confused with visual markers [3] which is only one false mental model that users have developed. According to Mäkelä et al. [14], who have analyzed how users perceive mobile interaction with NFCtags, mental models include text messaging, communication via Bluetooth or calling a specific number. Complementary, O’Neill et al. [17] showed that users were not sure whether to hover, slide, wave or press a tag with their mobile devices. Other difficulties with NFC-based interactions concern the correct alignment of NFC-units in mobile devices and tags on physical objects [8]. Furthermore, users often do not know how to initiate the interaction or are unsure about its course [4].

Several approaches have tried to improve the visual design and the usability of NFC-based mobile interaction: Arnall [2] has developed a graphic language to visualize NFC and the touch-based interactions with it. For example, he suggests using a circle with a dashed line to hint at the hidden functionalities of NFC-tags. Välkkynen et al. [25] have designed different symbols to visualize physical hyperlinks. The designs combine icons for different actions, e.g. connecting, information or download, and different selection methods, e.g. touch, scan or point, to create compound symbols. Riekki et al. [18] differentiate between different kinds of tags - general tags that identify tagged objects and special tags that also represent additional information or actions. A user study showed that 
the special tags were preferred as they hinted at the action to be performed. Häikiö et al. [11] designed a menu card for elderly people who could order meals for home delivery by touching NFC-tags on the menu. The design of the physical UI paid attention to the cognitive and physical challenges of elderly people, e.g. it used a large font and different colors to differentiate the tags. The interaction design took advantage of the simple interaction with NFC to avoid interaction with the tiny keypad or the screen of the mobile device. Broll et al. [3] suggest different designs to increase the learnability and guidance for mobile interaction with NFC, including dedicated start-tags and visual cues on physical UIs.

In order to investigate the visual design of physical, NFCbased UIs, we followed a user centered design process and made use of different prototyping techniques throughout this paper: Synder [23] demonstrated how ideas can be developed effectively through paper prototyping. Guerrilla HCI methods as mentioned by Nielsen et al. [16] are labor saving and cost effective practices. These opportunistic design methods and processes provide instruments that put users center stage [24] and drive the development phases.

In the following sections, we exemplify how the user centered design process was adopted and executed to refine and evaluate the visual design of tags and physical UIs for NFC-based mobile interaction.

\section{DESIGN PROCESS}

The development and evaluation of low- and high-fidelity prototypes for the visual design of NFC-based mobile interactions that we describe in this paper, was practiced through a user centered design process [15, 20], that was diverted into different phases (Figure 2) in order to receive a satisfying and usable end result.

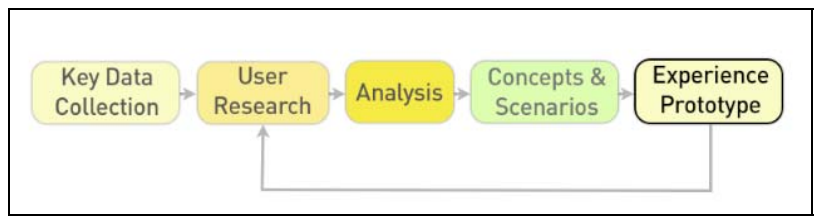

Figure 2. The user centered design process and its phases which were repeated iteratively throughout the design process.

During the phase of Key Data Collection, we gathered material from related work that is relevant for our own approach to the visual design of physical UIs (see previous section). Next, we collected data in early interviews during the User Research-phase and discussed our observations during the Analysis-phase in order to identify the main user needs regarding NFC-based mobile interaction:

- A unified visual design language to avoid confusion and to establish a common mental model for the interaction with NFC

- The accentuation of tagged objects and single tags as interactive elements
- Guidance for the basic interaction process, e.g. touching a tag correctly with a mobile device, especially for novice users

- The visualization of different actions, functionalities or information tags can be associated with

- Visual guidance during the interaction

In the Concept-phase we have built our ideas on top of these findings using different ideation techniques and created numerous design ideas for the different interaction phases (see next section). The best ideas were voted out and transferred into a scenario [7] which was prototyped on both high and low fidelities iteratively (see later sections). We developed a big variation of low fidelity prototypes [24] to explicitly invite users to bring in their feedback. In this phase we deliberately left the design direction very broad and open. With the Experience Prototypes [5] we stayed longer on a lower fidelity and its advantages [19] such as the focus on pure functionality rather than confronting users too early with design elements that would lead to unwanted feedback. In the first iterations, this was useful to narrow the scope down from the large amount of mockups to the main visual clues and symbols we wanted to provide.

The next section gives a brief overview of the different phases of mobile interaction with NFC, along which we have developed different low- and high-fidelity prototypes. After that, we explain how we carried out the different steps of the design process. We describe the iterations we have performed with symbols from the different interaction phases with low-fidelity prototypes in order to extract successful designs for the subsequent high-fidelity prototyping.

\section{PHASES OF INTERACTION}

In order to elicit further design requirements and to get a better understanding of the NFC-interaction process, we divided it into five distinct phases. This differentiation also helped us to explore the needs of experienced and novice users and to determine which visual designs can support the different steps of the interaction process best.

- Awareness: At first, the visual design of physical UIs tries to catch the interest of passers-by and make them aware of their interactive features. Visual designs for this phase may establish a trademark design for NFCbased interaction and thus increase the recognition value of NFC. They can help novice users to identify tagged objects, while experienced users may remember them faster.

- Approach: Once their attention is caught, users can approach a tagged object and learn how to interact with the physical UI and its tags from explanatory text or visual cues. This phase can be skipped by experienced users, but is a good way to explain the interaction to novice users in general and help them to overcome their initial inhibition threshold. 
- First Contact and Selection: The general approach is followed by the first contact with specific tags on the physical UI and their selection in order to carry out specific tasks. In this phase, it is important to visualize the individual information, features and functionalities that are associated with tags and are triggered when touching them. This phase should allow quick interaction for experienced users, but also provide explanation and guidance for inexperienced users.

- Completion: As the final step of the interaction process provides closure, it usually comprises critical interactions, e.g. payment or submitting data to a service. Visualizations should point out the impact of this interaction on the whole process in order to emphasize its criticality.

- Dropout: The interaction process can be interrupted at any time, either by leaving the physical UI or by explicitly aborting the interaction on the mobile device or by touching a tag on the physical UI. This phase is crucial as it establishes a high level of trust between users and the system, related to Nielsen's heuristic [16] of user control and freedom.

\section{LOW-FIDELITY PROTOTYPES}

In order to receive some first feedback about which design direction might be the most usable and successful we first brainstormed on appropriate metaphors that would lead the user towards a successful interaction. After the ideation phase, which was mainly executed using the creative thinking method mentioned by Buxton [6] we created a first set of 20 early prototypes for each of the individual interaction phases (see previous section). Ideas were turned into symbols that would match a certain section of the whole experience. Some concepts for example were focusing on the invisible nature of NFC-tags; the graphical solution incorporated an aura (Figure 3) of dotted lines in order to raise the guidance on where to touch the area of the tag. These concepts are based on Arnall's [2] graphical language for NFC-based interactions.

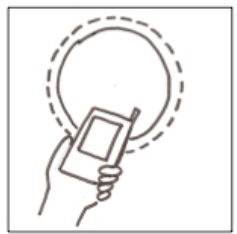

a)

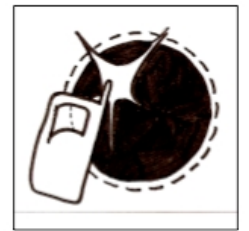

b)

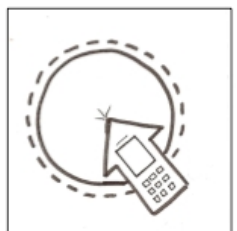

c)
Figure 3. Variations of low-fidelity Explanatory Symbols that explain the interaction with NFCtags during the First Contact-phase

We have extended parts of the visual language, creating elements that include various shapes, of both abstract and geometrical nature (Figure 4c) in order to offer a big spread of designs to testers. We created adequate symbols on a low fidelity to invite users to bring in their feedback and own ideas in the first place. The visual design of these symbols varies according to the kind of assistance they provide during one of the different interaction phases they are associated with. For the Awareness-phase, we created Adhesive Symbols to catch the attention of potential users and to imply that interaction with NFC is possible in this context (Figure 4). Building on the fact that until today there is no common standard for a global brand signage for NFC we combined existing symbols such as the already familiar NFC logo from Nokia ${ }^{5}$ (Figure 4b) with new visual designs. The same methodology was applied to lowfidelity prototypes of Explanatory Symbols for the First Contact-phase, which are intended to explain the concept of touching tags with a mobile device (Figure 3).

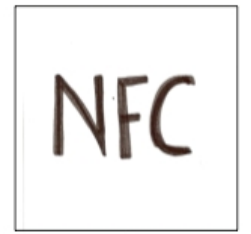

a)

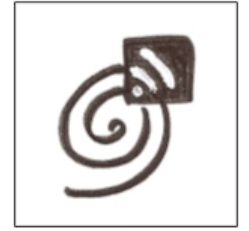

b)

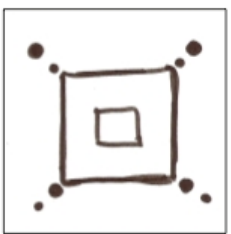

c)
Figure 4. Early low-fidelity Adhesive Symbols to catch the attention of users during the Awareness-phase

Different Action Symbols were developed for the Selectionphase in which users can trigger certain actions such as shown in Figure 5. These symbols were inspired by GUI elements that might have an advantage of familiarity and would imply the hidden action directly, such as picking up the phone or opening the help menu for example.
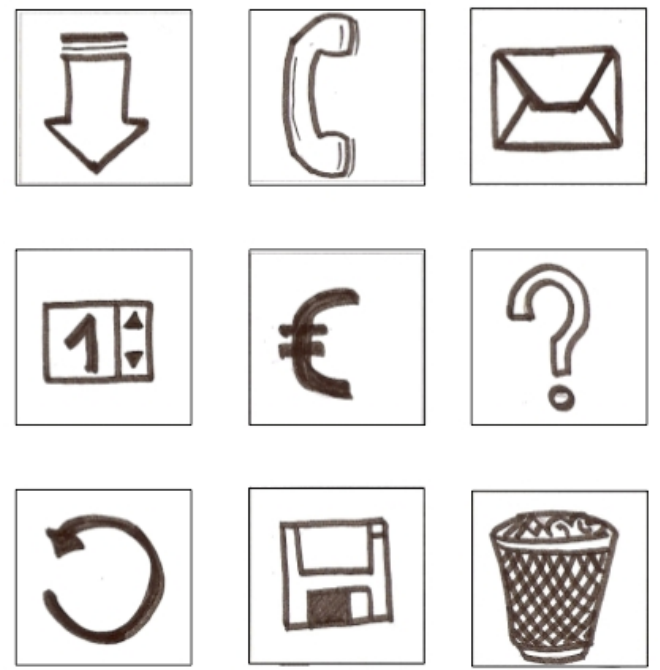

Figure 5. Different Action Symbols to visualize features associated with NFC-tags during the Selection-phase

After designing various symbols for the different interaction phases with paper prototypes, we conducted an online survey to investigate the preferences of potential users, to reduce the number of designs to the most favorite ones and to gain further insights about their usability and appropriateness. The online questionnaire was completed 
by 166 subjects (90 male, 76 female) with an average age of 26, ranging from 18 to 45 . Most subjects were students and had different educational backgrounds, ranging from computer sciences and math to medicine, politics and literature. 49 of them had prior experiences with NFCbased mobile interaction, e.g. from previous user studies, whereas 117 subjects had no such experiences.

We asked the subjects which 3 icons of our designs they considered to be the most, respectively the least appealing ones regarding the interaction with NFC. After measuring and adopting the results we got rid of most of the initial designs and concluded this session with 3 sets of icons (Figures 3, 4, 5). We used the resulting low-fidelity icons as a basis for the design of high-fidelity prototypes, which are presented in the next section.

\section{HIGH-FIDELITY PROTOTYPES}

Based on the findings of the preliminary online survey, we have designed high-fidelity prototypes for an advertisement scenario. We chose an advertisement poster as a physical UI, since posters provide a good way to evaluate the composition of visual elements like Adhesive, Explanatory or Action Symbols (Figures 3, 4, 5), as well as their supporting influence on users during the interaction.

Altogether, there are five different designs of an advertisement poster for a museum exhibition, which can be used to order tickets for a guided tour through NFCbased mobile interaction (Figures 6 to 11). In general, we decided to set the advertisement into focus in order to maintain the characteristics of an advertisement. Therefore, all designs are based on the same application structure and provide the same application features either on the physical UI or on the mobile device. On the other hand, the designs vary in different details regarding the layout and the visual design of the physical UI as well as the interaction with its NFC-tags. Each high-fidelity prototype and the concept behind it will be explained in the following sections.

\section{Design A}

Design A works with a reduced number of NFC-tags (Figure 6). At the bottom left, a toolbar provides general features, e.g. a help-function to which users may refer to when encountering difficulties during the interaction. Furthermore, users have the possibility to access general information about the exhibition or visit its homepage by touching the corresponding symbols in the toolbar.

In order to purchase tickets, the design uses only one Explanatory Symbol that combines different designs from the preliminary paper prototyping for several purposes: Firstly, it is intended to catch the attention of users and to evoke their curiosity (Awareness-phase). Secondly, the symbol explains the interaction with the physical UI (Approach-phase) and thus plays an important role during the first contact with the poster. The Explanatory Symbol is four times bigger than regular NFC-symbols on the physical UI to increase its awareness. For a better understanding, we added text that informs users about the interactive features of the advertisement and hints at the further procedure that users have to expect when touching the symbol with their mobile device. This helps to decrease the inhibition threshold of novice users and may be easily skipped by expert users.

In order to initiate the ticket purchase, users have to touch the Explanatory Symbol. Regarding the NFC-interaction, this is the only contact between the physical UI and the mobile device. The remaining steps of the interaction are carried out on the latter. Users may also abort the interaction at any time by selecting the corresponding function on the mobile device. The advantage of this approach is the reduction of attention shifts between the physical UI and mobile devices. It also highlights the characteristics of the advertisement, whereas only little space is spared for NFC-tags.

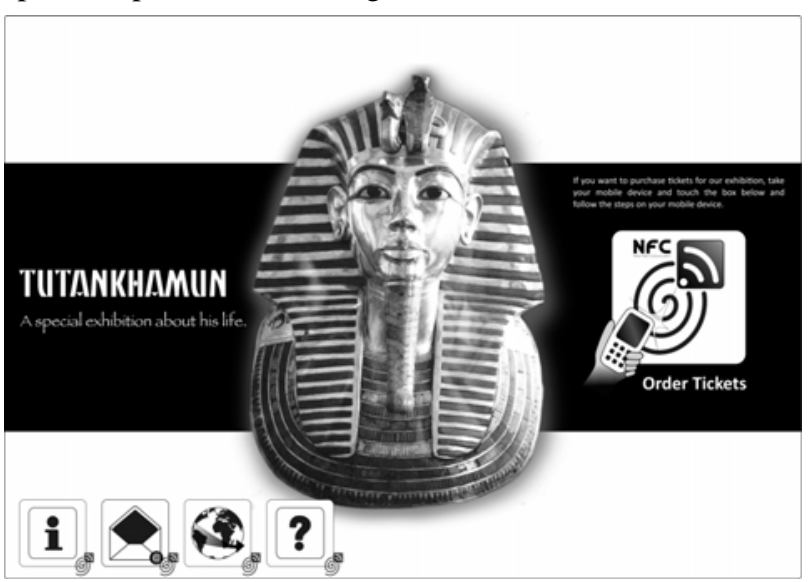

Figure 6. Design A with a single symbol to explain the interaction with the NFC-tag and to initiate the further interaction on the mobile device

\section{Design B}

Design B works with a transparent layer on the right side of the poster to separate advertisement and interactive space (Figure 7). This facilitates the arrangement of visual and interactive components and emphasizes the NFCinteraction without stealing the advertisement's focus.

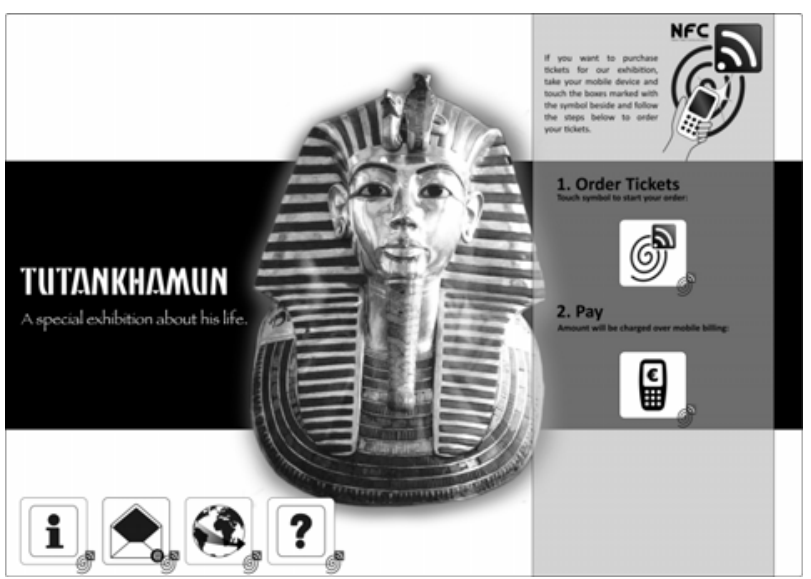

Figure 7. Design B with two NFC-tags to start and finish the interaction with the ticketing service 
The top of the transparent layer is used for the Awarenessphase and the Approach-phase. Therefore, an Explanatory Symbol is used to catch the attention of potential users and to explain the interaction at the same time. For novice users, additional text next to the symbol describes the interaction in more detail. The remaining space is used for the purchase of tickets.

The interaction process is split into three steps and maps crucial actions to tags on the physical UI. In the first step users make contact with the physical UI and initiate the interaction by touching the first tag. In the next step, the further interaction continues on the mobile device (Figure 8a). For the third step, users are asked to shift their attention back to the poster and touch the second tag with their mobile devices, in order to pay for the tickets and thus complete the interaction (Figure 8b). The top down arrangement supports the interaction workflow and indicates the order of selection. Numbered headlines with brief explanations describe the actions to be triggered and give an overview of the steps of the interaction. The advantage of this design is the physical closure of the interaction, since the interaction starts and ends on the poster, reinforcing the feeling of having completed the interaction successfully.

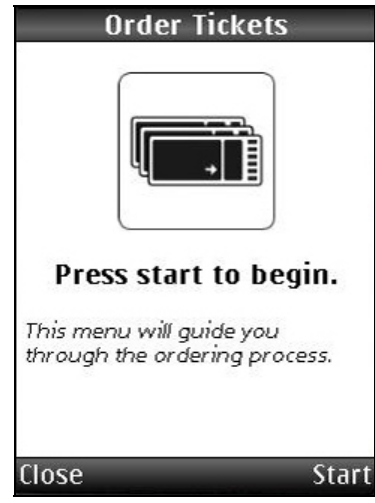

a)

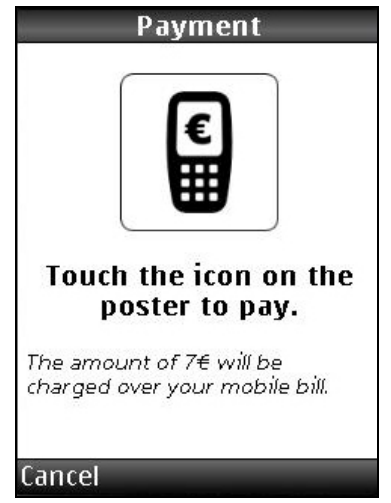

b)
Figure 8. Screens of the mobile application for ordering tickets (a) and finishing the interaction (b)

\section{Designs C and D}

Designs C and D (Figures 9 and 10) are similar to the preceding design, but divide the interaction process into three different steps, providing dedicated Action Symbols on the physical UI for each interaction - one to select tickets, one to add a guided tour and one to complete the payment and the interaction. That way, users can easily get an overview of the steps of the Selection-phase. The Action Symbols can be seen as entry points to associated application features, which are carried out on the mobile device. For example, when users touch the ticket-symbol on the physical UI, a corresponding screen appears on the mobile device, where users can input the number of tickets they would like to order.
In comparison to design $\mathrm{C}$, design $\mathrm{D}$ works with additional Action Symbols to describe the kind of selection (Figure 10). Two Action Symbols are placed next to each other. The first symbol is a description of the action and not interactive, whereas the second symbol is marked with an Adhesive Symbol to show its interactivity. The symbols have to be read from left to right. Each combination of symbols stands for a phrase and describes the functions that are hidden behind the second Action Symbol.

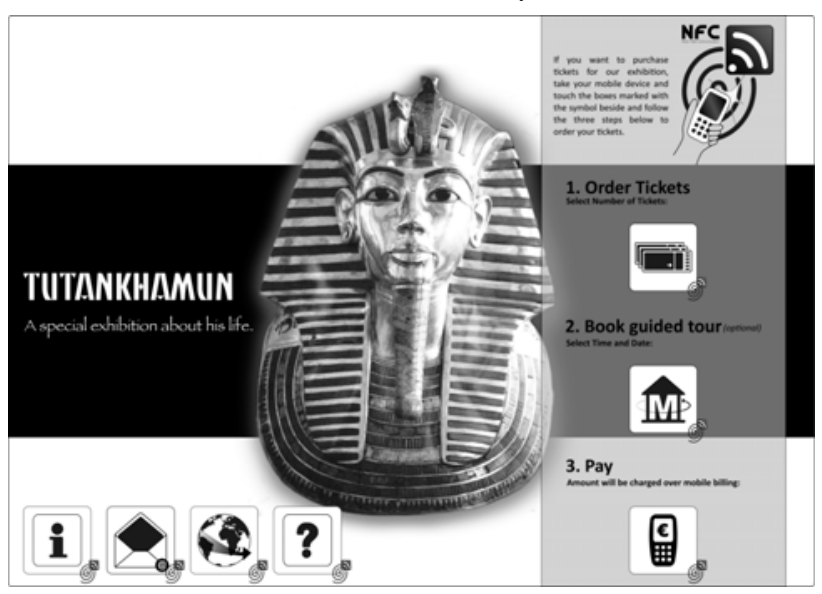

Figure 9: Design C with dedicated Action Symbols for the different steps of the Selection-phase

The first combination visualizes the phrase 'to order tickets for the exhibition, select the number of tickets', whereas the second combination states 'for an additional guided tour, select the time and date'. Thus, the first symbol may be considered as a headline, whereas the second symbol offers further details about the selection on the mobile device. However, this approach is skipped for the action "Pay" to create a break in the interaction workflow and to highlight the criticality of this action. This will help novice users to notice the importance of this action, and will avoid unintended and habitual confirmations by expert users.

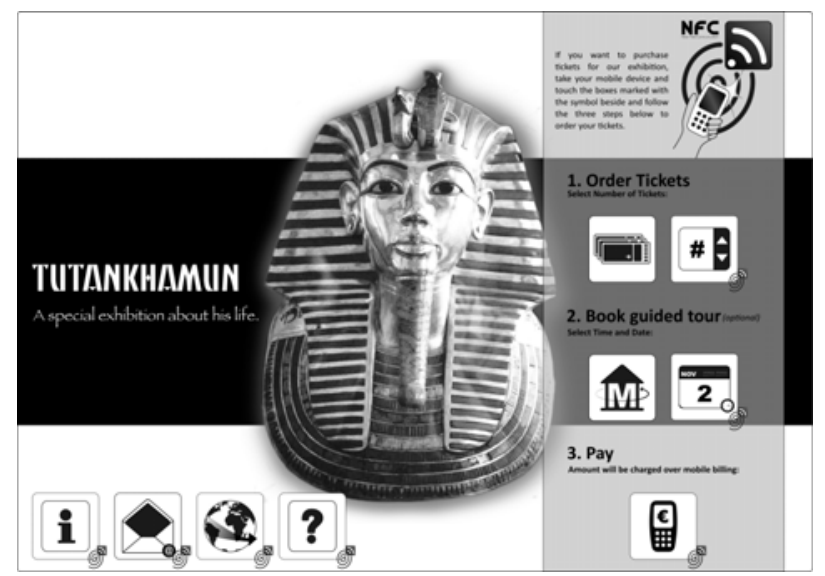

Figure 10. Design D with additional Action Symbols to visualize the interaction steps in more details 


\section{Design $\mathrm{E}$}

Design E comprises a larger interaction space and allows users to carry out most of the selections on the physical UI (Figure 11). Its layout is similar to the previous designs. The top of the transparent layer is used for the phases of Awareness and Approach, whereas the center focuses on First Contact and Selection, followed by Completion at the bottom. Action Symbols on the left side are not interactive and describe the type of selection. This will help expert users to skip reading the numbered headlines. A quick glance at the Action Symbols is sufficient and expert users may focus on their selection on the right side, where available options are listed and marked as interactive elements. If users don't find an option on the physical UI, they may select the "more"-option to get a list with further options on the screen of the mobile device.

In comparison to the remaining Action Symbols, the symbol for "Pay" is interactive and centered at the bottom of the transparent layer to emphasize its importance. The advantage of this design is the possibility to complete most selections on the physical UI, since most options are immediately available. Furthermore, it enables users to not only see the interaction steps that have to be carried out during the interaction, but they may also overview the options that are provided for each interaction step.

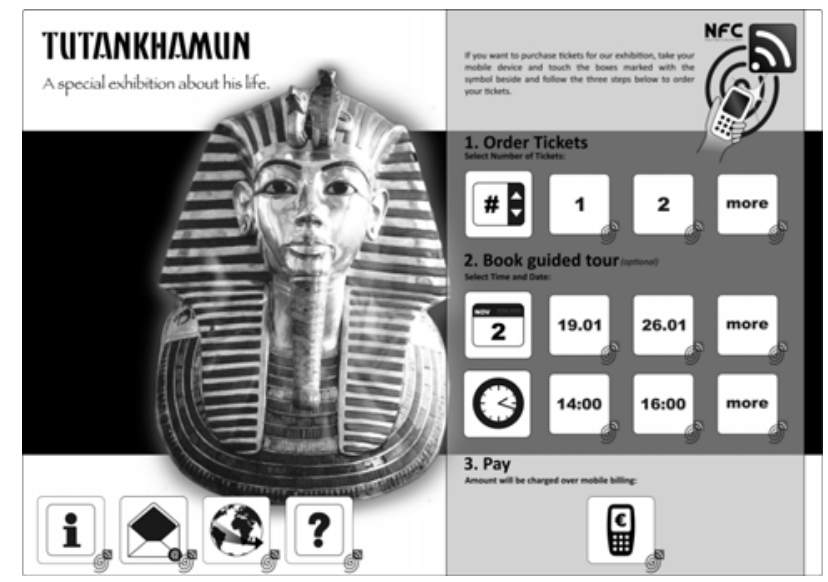

Figure 11. Design E maps most application features and options to NFC-tags on the physical UI

\section{USER STUDY}

\section{Setup and Experimental Design}

In order to evaluate the high-fidelity prototypes, we conducted a user study over a period of three days. The goal of the study was to identify user preferences, problems during the interaction as well as advantages and disadvantages of each high-fidelity prototype. Therefore, the study focused on qualitative evaluation. It lasted about 60 minutes for each subject and was recorded on video. At the beginning of the study, we informed the subjects about the procedure of the study and gave a short introduction to the mobile device (Nokia 6131 NFC) to explain the key functions and the location of the NFC-reader.
During the study, the subjects had to carry out a task for which they had to interact with the different designs of the advertisement poster to buy two tickets with a guided tour for a specific time and date (Figure 12). This task had to be completed with all five high-fidelity prototypes. In order to avoid learning effects, the order of the designs was randomized, using a Latin Square design. The scenario ended with a post-scenario questionnaire, where users had to choose the designs they liked the most and the least. Furthermore, the subjects had to fill out a questionnaire for each design, regarding the aesthetical appeal as well as the usability aspects of the physical UI. The questionnaire was based on the IBM Computer Usability Satisfaction Questionnaire [13] and used a 5-point Likert-scale from 1 ("strongly disagree") to 5 ("strongly agree") to let the subjects rate different statements about the designs.

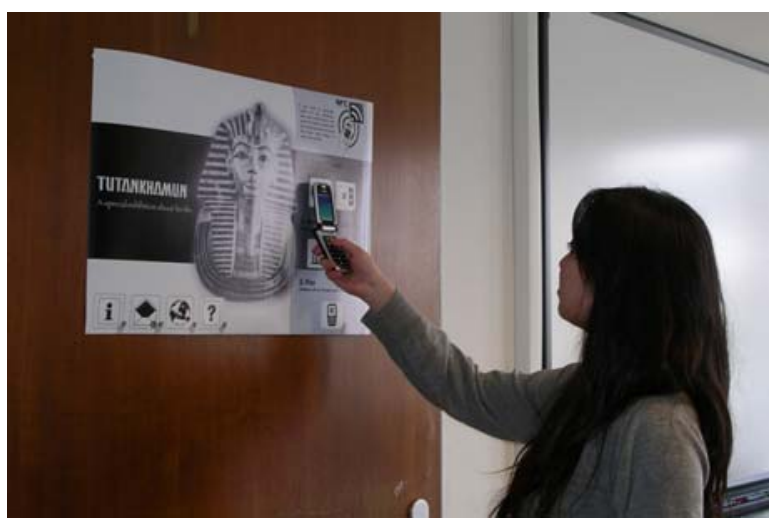

Figure 12: A subject interacting with a tagged poster

\section{Results}

The study was conducted with 21 subjects (17 males and 4 females) with an average age of 24 (ranging from 19 to 30 ). Most of them were students or graduates in computer science. Some of them studied or graduated in chemistry, finance or literature. Regarding their prior experience with NFC-based mobile interaction, only 6 subjects stated to have previous knowledge, whereas 15 of them did not.

Design $\mathrm{A}$ is the advertisement that the subjects liked the most and is thus ranked on the first place with 9 votes, followed by design $\mathrm{B}$ with 5 and design $\mathrm{C}$ with 4 votes. In comparison to this, designs $\mathrm{D}$ and $\mathrm{E}$ are on the last ranks and are the designs that the subjects liked the least, with 6 and 10 votes. The reasons that the subjects gave for their ratings were quite similar: Design $\mathrm{A}$, for example, was mostly favored because of its simple interaction which required only little interaction with the physical UI, whereas the remaining part of the task could be completed on the mobile device which users felt more comfortable with. Furthermore, the design required less attention shifts between the physical UI and the mobile device. Besides the simple interaction, the subjects also liked the visual appearance of the physical UI, which was described as simple to use, clear and trustworthy. Nevertheless, not all subjects rated the design as useful. Some criticized that the advertisement contained too little information about the 
exhibition, e.g. dates and time slots for the guided tour. Also, some subjects did not like the few options and would have preferred more interaction with the physical UI, wherefore they voted for designs B or C.

Design B is ranked second with 5 votes and differs from design A in only two aspects. Instead of one NFC-tag, design $B$ has two tags to initiate and to finish the interaction. Furthermore, the design provides a brief overview of the interaction steps. These two characteristics are the reasons for the subjects' choice, since they found it important to confirm the ticket payment explicitly. They also rated the physical UI as clear and easy to understand.

Subjects who voted for design C liked the appearance of the advertisement and the clear and neat structure of the physical UI. Another reason was the division of the interaction process into three steps and their representation by three corresponding Action Symbols on the physical UI. The subjects appreciated the possibility to grasp the most important interaction steps at one glance and liked the clear separation between selection of tickets, guided tour and payment. They also liked the balanced interaction between physical UI and mobile device.

Whereas the subjects rated designs A - C positively, designs $\mathrm{D}$ and $\mathrm{E}$ received more negative reviews and are thus found on the lower ranks. The main reason for the selection of design $\mathrm{D}$ as one of the least favorite designs was the usage of two combined Action Symbols to describe one action. Subjects were confused and did not know which symbol they should interact with, resulting in a more complicated interaction.

Design $\mathrm{E}$ has received even more negative votes and is ranked last. Although the subjects appreciated the quick selection of options on the physical UI, the benefit from such direct selection to complete the task was estimated as rather low, since not all options were available on the physical UI. Due to the large number of NFC-tags on the advertisement, subjects needed time to orient themselves on the UI, resulting in a rather negative rating for the visual appeal of the advertisement. Some subjects described the design as confusing, complicated, overloaded and intimidating. Furthermore, they criticized the number of attention shifts between physical UI and mobile device and named it as one of their reasons to dislike the design.

These statements were also supported by the ratings that the subjects had given for the post-scenario questionnaire. Figure 13 presents an overview of these ratings. In general, the three best liked designs had achieved better ratings than the designs on the last two ranks. While the subjects considered designs $\mathrm{A}, \mathrm{B}$ and $\mathrm{C}$ as aesthetically appealing, designs $\mathrm{D}$ and $\mathrm{E}$ received the worst ratings, since information on the physical UI was badly structured and thus hard to find. Additionally, the subjects rated design D as rather difficult to understand, found it unclear on how to start the interaction on the physical UI, but agreed that it was easy to learn how the interaction works. With regard to the ease of learning, subjects thought that design A was the easiest to learn, closely followed by designs B and C.

At the end of the post-scenario questionnaire, the subjects also had to answer which kind of interaction they preferred to accomplish the given tasks. 12 of them preferred the combination of physical UI and mobile device, 5 of them preferred the mobile device and its regular UI and 3 subjects preferred the physical UI. For one subject, it did not matter at all. Subjects who preferred the mobile device to carry out the interaction, felt more familiar and comfortable with a mobile device out of habit.

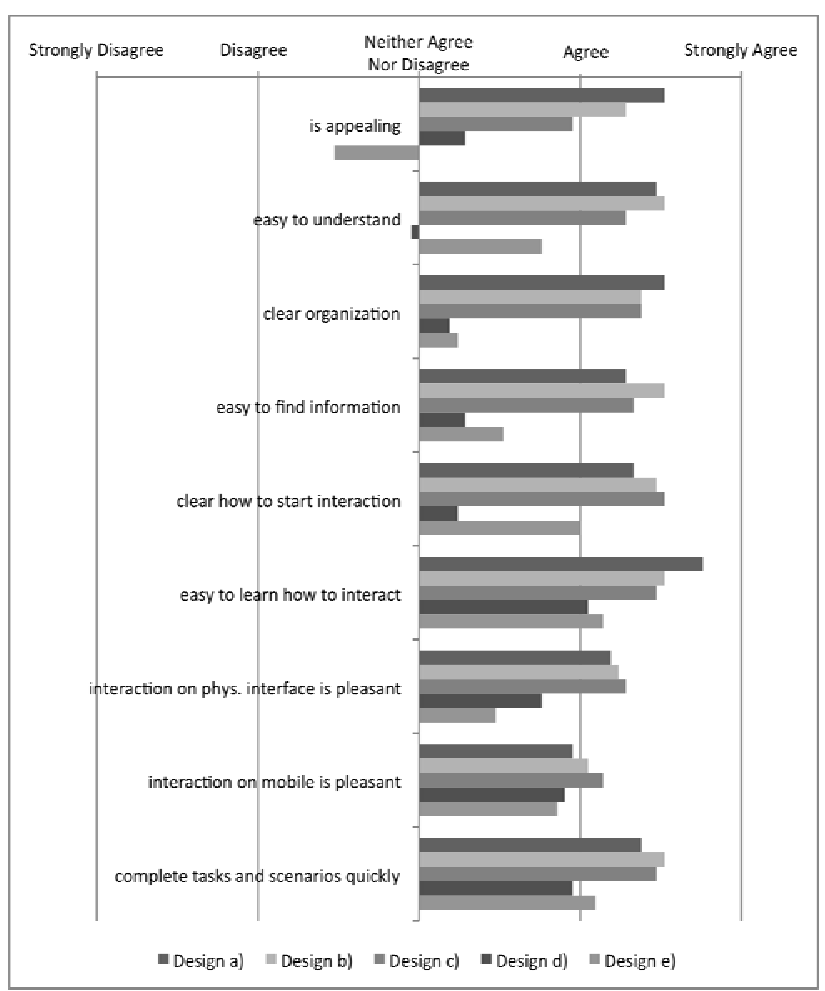

Figure 13. Overview of ratings for designs A - E

Nonetheless, most subjects preferred the combination of physical UI elements and a mobile device, since they found it easier to start the interaction by touching the NFC-tags on the physical UI, but preferred to concretize their selection on the mobile device. They also appreciated the idea to complete critical actions on the physical UI to avoid unintended confirmation. However, opinions about this aspect differed. 8 preferred the confirmation on the mobile device, 7 preferred it on the physical UI, and 6 would like to be able to confirm on both components. We asked the subjects, where they would like to perform the switching between parts of the application. The opinions differed and 8 of them liked to complete it on the physical UI, whereas 7 would like to do it on the mobile device.

The video recording of the subjects allowed different observations during the interaction with the given highfidelity prototypes. 2 out of 21 subjects were not able to start the task with the first design they were given. Both of 
them seemed to be inhibited to interact with the physical UI, but after showing them how the interaction worked, they could imitate it to complete their tasks. The remaining subjects had no major difficulties, but quickly learnt how to interact with the physical UI and became better with each design. At the beginning, some subjects had difficulties to read the content of the NFC-tag because they held the mobile device too far away, touched the NFC-tag for too short a period of time or did not correctly align the reading unit of the mobile device. Therefore, some subjects even used their hands to feel whether there was an NFC-tag hidden beneath the physical UI or not. Few subjects were unsure whether the mobile device could be removed from the NFC-tag after touching and kept it close to the NFC-tag to make their input. However, after a few trials with different designs they figured out that it was not necessary to keep it close to the NFC-tag.

In general, the subjects learnt quickly how the interaction worked and became better with each design. Many of them used the provided help function when not sure on how to continue or where to begin. The time required before making the first contact with the physical UI varied from subject to subject and ranged from 2 to 42 seconds. The short time participants spent for the Awareness Phase and Approach Phase may indicate that explanatory text is just scanned but not consciously read.

\section{DISCUSSION AND CONCLUSION}

The results of the user study have shown that the subjects prefer advertisements that are kept simple with regard to the interaction with NFC, meaning that the number of NFC-tags on the physical UI should be reduced in order to design a clear UI that appeals to users. The interaction should be started by touching an NFC-tag on the physical UI, because subjects regarded it as convenient and fast.

Regarding the following interactions, the opinions were ambiguous. Some subjects preferred to switch between parts of the application by touching NFC-tags, others felt more comfortable to complete this on their mobile devices, since they were more used to it. Therefore, the design of the following interaction is dependent on the application. If the estimated length of the selection phase is rather long, a touch-and-go approach may be advisable, as users do not have to remain in front of the advertisement. If few selections have to be made, the physical UI should provide corresponding NFC-tags in order to give an overview of the interaction steps to guides users through the interaction process. In this case, the confirmation of critical actions should also be shifted to the physical UI to avoid unconscious confirmations, whereas the mobile device should be used to concretize the selection of options, like the input of a number of tickets or the selection of a specific date.

Action Symbols on the physical UI should be interactive, since users have constituted the association between Action Symbols and NFC-tags and did not pay attention to the Adhesive Symbol at the corner. Therefore, the use of Action
Symbols as descriptive components may lead to confusions and should be avoided. Action Symbols are also a good mean to provide guidance on the mobile device. Users found it helpful to see the next Action Symbol on the screen of the mobile device, so that they could touch the corresponding symbol on the physical UI.

The given conclusions are an initial attempt to point out important aspects regarding the design of physical UI and should inspire further work in this area. This is essential in order to establish design recommendations for physical UI for NFC. Once this is done, the interaction will become easier and the spread of NFC may increase.

\section{REFERENCES}

1. ABI Research. 2007. Twenty Percent of Mobile Handsets Will Include Near Field Communication by 2012.www.abiresearch.com/abiprdisplay.jsp?pressid=8 38

2. Arnall, T. 2006. A Graphic Language for Touch-based Interactions. In Proc. of MIRW 2006, Espoo, Finland.

3. Broll, G., Keck, S., Holleis, P., and Butz, A. 2009. Improving the accessibility of NFC/RFID-based mobile interaction through learnability and guidance. In Proc. of MobileHCI'09 (Bonn, Germany, September 15 - 18, 2009). ACM, New York, NY, 1-10.

4. Broll, G., Rukzio, E., Paolucci M., Wagner, M., Schmidt, A., and Hussmann, H. 2009. Perci: Pervasive Service Interaction with the Internet of Things. In IEEE Internet Computing 11/12 2009, IEEE Computer Society, 2009, 74 - 81.

5. Buchenau, M. and Suri, J. F. 2000. Experience prototyping. In Proc. of DIS'00 (New York City, New York, United States, August 17 - 19, 2000). D. Boyarski and W. A. Kellogg, Eds. DIS '00. ACM, New York, NY, 424-433.

6. Buxton, B. 2007. Sketching User Experiences: Getting the Design Right and the Right Design. Elsevier 2007

7. Carroll, J. M., Ed. 1995 Scenario-Based Design: Envisioning Work and Technology in System Development. John Wiley \& Sons, Inc.

8. Geven, A., Strassl, P., Ferro, B., Tscheligi, M., and Schwab, H. 2007. Experiencing real-world interaction: results from a NFC user experience field trial. In Proc. of MobileHCI '07, vol. 309. ACM, New York, NY, 234-237.

9. Hardy, R. and Rukzio, E. 2008. Touch \& interact: touch-based interaction of mobile phones with displays. In Proc. of MobileHCI '08. ACM, New York, NY, 245254.

10.Hartmann, B., Doorley, S., and Klemmer, S. 2008. Hacking, Mashing, Gluing: Understanding Opportunistic Design. IEEE Pervasive Computing, vol. 7, no. 3, pp. 46-54, July-Sept. 2008.

11.Häikiö, J., Wallin, A., Isomursu, M., Ailisto, H., Matinmikko, T., and Huomo, T. 2007. Touch-based 
user interface for elderly users. In Proc. of MobileHCI '07, vol. 309. ACM, New York, NY, 289-296.

12. ITU Internet Reports 2005: The Internet of things (ITU 2005 7th edition).

13.Lewis, J. R. 1995. IBM computer usability satisfaction questionnaires: psychometric evaluation and instructions for use. Int. J. Hum.-Comput. Interact. 7, 1 (Jan. 1995), 57-78.

14. Mäkelä, K., Belt, S., Greenblatt, D., and Häkkilä, J. 2007. Mobile interaction with visual and RFID tags: a field study on user perceptions. In Proc. of CHI '07. ACM, New York, NY, 991-994.

15. Moggridge, B. Designing Interactions. MIT Press 2006.

16. Nielsen, J. Guerrilla HCI: Using Discount Usability Engineering to Penetrate the Intimidation Barrier. Engineering. 1994:1-18.

17. O'Neill, E., Thompson, P., Garzonis, S., and Warr, A. 2007. Reach out and touch: Using nfc and 2d barcodes for service discovery and interaction with mobile devices. In Proc. of Pervasive'07, volume 4480 of Lecture Notes in Computer Science, 19-36. Springer, 2007.

18. Riekki, J., Salminen, T., and Alakarppa, I. 2006. Requesting Pervasive Services by Touching RFID Tags. IEEE Pervasive Computing 5, 1 (Jan. 2006), 40.
19.Rudd, J., \& Stern, K., \& Isensee, S.: Low vs. highfidelity prototyping debate. Interactions. ACM Press 1996.

20. Saffer, D. Designing for interaction. New Riders. 2006.

21.Sánchez, I., Riekki, J., and Pyykknen, M. 2008. Touch \& control: Interacting with services by touching RFID tags. In Proc. of IWRT 08, June 12-13 2008.

22.Schwieren, J. and Vossen, G. 2007. Implementing Physical Hyperlinks for Mobile Applications Using RFID Tags. In Proc. of IDEAS`07. IEEE Computer Society, Washington, DC, 154-162.

23. Snyder, C. Paper prototyping: the fast and easy way to design and refine user interfaces. Morgan Kaufmann 2006.

24.Svanæs, D. \& Seland, G.: Putting the Users Center Stage: Role Playing and Low-fi Prototyping Enable End Users to Design Mobile Systems. In Proc. CHI, 2004.

25.Välkkynen, R., Tuomisto, T., and Korhonen, I. Suggestions for Visualising Physical Hyperlinks. In: Permid. Munich, Germany, 2005, pp.35-38.

26. Want, R., Fishkin, K. P., Gujar, A., and Harrison, B. L. 1999. Bridging physical and virtual worlds with electronic tags. In Proc. of CHI '99. ACM, New York, NY, 370-377.

27. Want, R. 2006. An Introduction to RFID Technology. IEEE Pervasive Computing 5, 1 (Jan. 2006), 25. 